\title{
Therapeutic effect of vascular interventional therapy and aspirin combined with defibrase on cerebral ischemia in rats
}

\author{
BAOSHAN LI ${ }^{1}$, SHOUKE GENG ${ }^{2}$ and YUANLI DA ${ }^{3}$

\begin{abstract}
${ }^{1}$ Department of Neurosurgery, The Third People's Hospital of Qingdao, Qingdao, Shandong 266041; ${ }^{2}$ Department of Neurosurgery, The Third People's Hospital of Pingdu City, Qingdao, Shandong 266753; ${ }^{3}$ Department of Neuro-Rehabilitation, The Third People's Hospital of Qingdao, Qingdao, Shandong 266041, P.R. China
\end{abstract}

Received February 20, 2018; Accepted May 30, 2018

DOI: $10.3892 /$ etm.2018.6271

\begin{abstract}
Therapeutic effect of vascular interventional therapy and aspirin combined with defibrase in the treatment of cerebral ischemia in rat model were investigated. Ninety rats were selected to establish cerebral ischemia model. Animal models were randomly divided into observation group and control group, with 45 rats in each group. Rats in observation group were treated with vascular intervention, and control group was treated with aspirin combined with defibrase. Peak systolic velocity (Vs) and end-diastolic velocity $(\mathrm{Vd})$ were compared between two groups before and after 12 months of treatment. Therapeutic effects were compared before and after 6 months, and before and after 12 months of treatment. Vs and Vd of vascular lesions in observation group at 12 months were reduced after treatment, and were significantly lower than those in control group $(\mathrm{p}<0.05)$. After treatment, Vs and Vd in observation group were significantly reduced $(\mathrm{p}<0.05)$. There was no significant difference in Vs and Vd values between the groups before treatment $(p>0.05)$. NDS scores in observation group were significantly lower than those in control group at 6 months and 1 year after treatment $(\mathrm{p}<0.05)$. There were no significant changes in NDS score at 6 and 12 months after treatment in control group compared with pretreatment group ( $p>0.05)$. NDS in observation group was significantly reduced at 6 and 12 months after treatment compared with pretreatment level $(\mathrm{p}<0.05)$. One year after treatment, incidence of cerebral infarction and transient ischemic attack and mortality in observation group were significantly lower than those in the control group $(p<0.05)$. Intracranial vascular interventional therapy can achieve satisfactory outcomes in the treatment of
\end{abstract}

Correspondence to: Dr Yuanli Da, Department of Neuro-Rehabilitation, The Third People's Hospital of Qingdao, Qingdao, 29 Yongping Road, Qingdao, Shandong 266041, P.R. China E-mail:di37qz@163.com

Key words: interventional therapy, aspirin, defibrase, cerebral ischemia cerebral ischemia, and can effectively promote nerve function recovery, and reduce the incidence of cerebrovascular diseases and mortality.

\section{Introduction}

Ischemic cerebrovascular disease (ICVD) is a disorder of blood supply to the brain based on the pathological changes of the blood vessel wall. Cerebral ischemia in blood supply area causes necrosis of the hypoxia, which in turn leads to local or diffuse brain damage, resulting in a series of neurological deficit syndromes $(1,2)$. With the economic development, improvement of people's living standard and acceleration of population aging, incidence of ICVD in China is gradually increasing. ICVD mainly includes cerebral infarction and transient ischemic attack. Mortality and disability rate of ICVD are high, seriously affecting human health and bringing financial burden to patients' families and society (3). The principle of ICVD treatment is to save lives, reduce brain damage, promote the recovery of nerve function, and prevent recurrence (4). Clinical treatment of this disease is mainly based on the drug treatment in order to achieve anticoagulant and antithrombotic effects, as well as stabilize plaques, but the outcomes of drug treatment are usually poor (5). Vascular interventional therapy, which can directly remove occlusion of blood vessels to promote blood supply return to normal, and induce vascular lumen remodeling and restore blood supply to brain tissue, is novel treatment of cerebrovascular disease (6). The application of endovascular stenting has been widely studied. A controlled clinical trial has also confirmed that this treatment method can effectively improve the prognosis of ICVD, and reduce the incidence of cerebrovascular events (7).

In this study, rat model of cerebral ischemia was established, and rats were randomly divided into vascular intervention group and aspirin combined defibrase drug treatment group. The peak systolic velocity (Vs), end-diastolic velocity (Vd), degree of neurological deficit and cerebrovascular disease events of the two groups were compared before and after the treatment to evaluate the therapeutic effect of vascular intervention in ICVD, so as to provide the basis for clinical treatment of ICVD. 


\section{Materials and methods}

Experimental animals. A total of 90 healthy male SD rats aged 49-56 days with body weight of 250-300 g were purchased from Animal Center of Guangzhou University of Chinese Medicine (Guangzhou, China). Rats were randomly divided into observation and control groups, 45 in each group. After the establishment of cerebral ischemia model, rats in observation group were treated with vascular intervention, and rats in control group were treated with aspirin combined with defibrase. Rats were housed in a temperature controlled room $\left(21 \pm 2^{\circ} \mathrm{C}\right)$ on a $12: 12 \mathrm{~h} \mathrm{light/}$ dark cycle (lights on at 06:00). All rats had free access to water and food. The study was approved by the Ethics Committee of The Third People's Hospital of Qingdao (Qingdao, China).

Establishment of rat cerebral ischemia model. After conventional anesthesia, rats were fixed and an incision was made on the neck skin. Common carotid arteries were separated. An incision was made on the middle region of posterior face to expose bilateral first cervical transverse femoral holes. Condensation of the vertebral artery was performed for 2-4 sec, and then permanent bilateral occlusion of the vertebral artery was done, and incisions were closed. After $24 \mathrm{~h}$, carotid arteries on both sides were closed with an artery clip for $20 \mathrm{~min}$, followed by reperfusion. Rats with subarachnoid hemorrhage, death or other pathological changes were excluded from the study.

\section{Method}

Vascular intervention. Before surgery, rats in observation group were subjected to gavage administration of aspirin enteric-coated tablets (300 mg/day; state approval no. H41023324; Kaifeng Baiyun Pharmaceutical Co., Ltd., Kaifeng, China) and clopidogrel bisulfate tablets $(75 \mathrm{mg} /$ day; state approval no. H20000542; Shenzhen Xinlitai Pharmaceutical Co., Ltd., Shenzhen, China) for 3 consecutive days. Experimental animals were anesthetized; the right femoral artery was punctured by Seldinger puncture; the $4 \mathrm{~F}$ arterial sheath was implanted; angiography was performed with a single bend angiography, and arterial circulation was observed. The $4 \mathrm{~F}$ micro-guidewire tip was inserted into the carotid artery, and was pushed to pass through the stenosis. Balloon dilatation at stenosis was performed, and stent was placed in the stenosis. Angiography was performed again to assess vascular circulation after stent implantation. Postoperative treatment with aspirin $(300 \mathrm{mg} / \mathrm{day})$ and clopidogrel sulfate tablets ( $75 \mathrm{mg}$ /day) was performed for 6 months, then changed to aspirin at a dose of $100 \mathrm{mg} /$ day.

Treatment using aspirin combined with defibrase. Control rats were subjected to a simple drug treatment program including clopidogrel sulfate $(75 \mathrm{mg} /$ day $)$ and aspirin $(300 \mathrm{mg} /$ day $)$ for 6 months, then changed to aspirin (100 mg/day). The drug used in control and observation group was produced from the same factory.

Observation index. The observation time of both groups was 12 months. Observation indexes included Vs and Vd before and after 12 months of treatment, incidence of cerebrovascular disease events (cerebral infarction, transient ischemic attack)
Table I. Comparison of general information between two groups.

\begin{tabular}{lccc}
\hline Groups & Male $(\%)$ & Weight $(\mathrm{g})$ & Age (days) \\
\hline $\begin{array}{l}\text { Observation } \\
(\mathrm{n}=43)\end{array}$ & $21(48.84)$ & $274.83 \pm 3.24$ & $54.00 \pm 0.28$ \\
$\begin{array}{l}\text { Control } \\
(\mathrm{n}=45)\end{array}$ & $23(51.11)$ & $276.59 \pm 3.71$ & $55.00 \pm 0.31$ \\
$\mathrm{t} / \chi^{2}$ & 0.045 & 1.151 & 0.892 \\
P-value & 0.831 & 0.653 & 0.112 \\
\hline
\end{tabular}

Table II. Comparison of Vs between two groups (mean \pm standard deviation, $\mathrm{cm} / \mathrm{sec}$ ).

\begin{tabular}{lcccc}
\hline Groups & Before treatment & After treatment & t-test & P-value \\
\hline $\begin{array}{l}\text { Observation } \\
(\mathrm{n}=43)\end{array}$ & $151.23 \pm 21.37$ & $112.57 \pm 23.41^{\mathrm{a}}$ & 10.877 & $<0.001$ \\
$\begin{array}{l}\text { Control } \\
(\mathrm{n}=45)\end{array}$ & $150.43 \pm 20.17$ & $148.32 \pm 24.92$ & 2.686 & 0.560 \\
$\mathrm{t}$-test & 0.878 & 8.495 & & \\
P-value & 0.281 & 0.001 & & \\
\hline
\end{tabular}

Vs, peak systolic velocity. ${ }^{\mathrm{a} C o m p a r e d}$ with before treatment; $\mathrm{P}<0.05$.

Table III. Comparison of Vd between two groups (mean \pm standard deviation, $\mathrm{cm} / \mathrm{sec}$ ).

\begin{tabular}{lcccc}
\hline Groups & Before treatment & After treatment & t-test & P-value \\
\hline $\begin{array}{l}\text { Observation } \\
(\mathrm{n}=43)\end{array}$ & $41.72 \pm 9.66$ & $20.17 \pm 8.01^{\mathrm{a}}$ & 14.217 & $<0.001$ \\
$\begin{array}{l}\text { Control } \\
(\mathrm{n}=45)\end{array}$ & $41.92 \pm 9.42$ & $38.28 \pm 10.48$ & 2.198 & 0.490 \\
t-test & 0.098 & 3.688 & & \\
P-value & 0.827 & $<0.001$ & & \\
\hline
\end{tabular}

Vd, end-diastolic velocity. ${ }^{\mathrm{a} C o m p a r e d}$ with before treatment; $\mathrm{P}<0.05$.

and mortality at 6 and 12 months after treatment. Zea longa 5 -point method was used to assess the neurological deficit score (NDS) before and after 6 and 12 months of treatment in each group (8). Zero points: No symptoms of neurological deficit; 1 point: Unable to fully extend the right forelimb; 2 points: Horner sign appears, turning right when crawling; 3 points: The body tilts to the right when crawling; 4 points: Unable to crawl. Higher NDS score indicated more severe neurological dysfunction in rats.

Statistical analysis. SPSS 13.0 software (SPSS, Inc., Chicago, IL, USA) was used for statistical analysis. Normal distribution quantitative data were compared by using t-test and ANOVA with SNK-Q test as a post hoc test. Intragroup comparisons were performed by using paired t-test and repeated measures ANOVA with Fisher's exact test as a post hoc test. Qualitative 
Table IV. Comparison of incidence rates of cerebrovascular events between two groups (n, \%).

\begin{tabular}{|c|c|c|c|c|c|c|}
\hline \multirow[b]{2}{*}{ Groups } & \multicolumn{2}{|c|}{ Cerebral infarction } & \multicolumn{2}{|c|}{ Transient ischemic attack } & \multicolumn{2}{|c|}{ Mortality } \\
\hline & 6 months & 12 months & 6 months & 12 months & 6 months & 12 months \\
\hline Observation group $(n=43)$ & $2(4.65)$ & $3(6.98)$ & $0(0.00)$ & $2(4.65)$ & $3(6.98)$ & $6(13.33)$ \\
\hline Control group $(n=45)$ & $4(8.89)$ & $10(22.22)$ & $1(2.78)$ & $11(23.91)$ & $6(13.33)$ & $17(36.96)$ \\
\hline$\chi^{2}$ & - & 4.059 & - & 6.842 & 0.968 & 6.136 \\
\hline P-value & $0.677^{\mathrm{a}}$ & 0.044 & $0.456^{\mathrm{a}}$ & 0.009 & 0.328 & 0.013 \\
\hline
\end{tabular}

andicates the use of Fisher's exact test for statistical analysis.

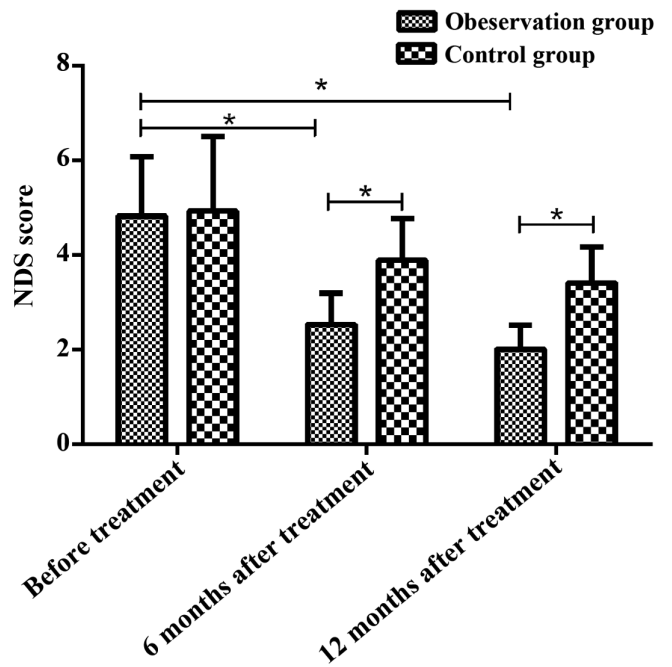

Figure 1. Comparison of neurological scores before and after treatment (points). There was no significant difference in NDS scores between the two groups before treatment $(\mathrm{p}=0.756)$. NDS scores in observation group were lower than those in control group at 6 and 12 months after treatment $(\mathrm{p}<0.05)$. There were no significant changes in NDS score at 6 months after treatment compared with that at 12 months after treatment in control group $(p>0.05)$. NDS score in the observation group was significantly reduced at 6 and 12 months after treatment compared with pretreatment values $(\mathrm{p}<0.05)$.

data were treated with $\chi^{2}$ test. Data with theoretical frequency $<5$ were subjected to Fisher's exact probability method. All P-values represented bilateral probability, and test level $\alpha$ was 0.05

\section{Results}

Comparison of general information between two groups. Two rats in observation group were excluded due to the failure in model construction, and 43 rats (male, $n=21$; female, $n=22$ ) with an average bodyweight of $274.83 \pm 3.24 \mathrm{~g}$ were finally included in subsequent analysis. A total of 45 rats (male, $n=23$; female, $\mathrm{n}=22$ ) with an average bodyweight of $276.59 \pm 3.71 \mathrm{~g}$ were included in control group. There were 21 males (48.84\%) in observation group and body weight was $274.83 \pm 3.24 \mathrm{~g}$. There were 23 males $(51.11 \%)$ in control group and body weight was $276.59 \pm 3.71 \mathrm{~g}$. There was no significant difference in male ratio and body weight between two groups $(\mathrm{p}>0.05)$ (Table I).

Comparison of Vs between two groups before and after treatment. There was no significant difference in Vs between observation group and control group before treatment $(p=0.281)$. After treatment, Vd value of observation group was lower than that of control group $(\mathrm{p}=0.001)$. After treatment, Vs value of observation group was significantly reduced $(\mathrm{p}<0.05)$, while no significant changes of Vs value in control group were observed ( $p>0.05)$ (Table II).

Comparison of Vd between two groups before and after treatment. There was no significant difference in Vd between observation group and control group before treatment $(\mathrm{p}=0.827)$. After treatment, $\mathrm{Vd}$ in observation group was significantly lower than that of control group $(\mathrm{p}<0.001)$. After treatment, Vd of observation group was significantly reduced $(p<0.05)$. After treatment, there were no significant changes in Vd in the control group ( $\mathrm{p}>0.05)$ (Table III).

Comparison of neurological deficits between two groups before and after treatment. There was no significant difference in NDS score between two groups before treatment $(4.82 \pm 1.25$ vs. $4.92 \pm 1.58 ; p=0.756)$. NDS score in observation group was significantly lower than that in control group at 6 months $(2.53 \pm 0.66$ vs. $3.88 \pm 0.89 ; \mathrm{p}<0.05)$ and 12 months $(2.01 \pm 0.51$ vs. $3.41 \pm 0.76 ; \mathrm{p}<0.05)$ after treatment. There were no significant changes in NDS score at 6 months after treatment compared with that at 12 months after treatment in control group ( $\mathrm{p}>0.05)$. NDS score in the observation group was significantly reduced at 6 and 12 months after treatment compared with pretreatment values $(\mathrm{p}<0.05)$ (Fig. 1).

Comparison of incidence rates of cerebrovascular events between two groups. The incidence of cerebral infarction, transient ischemic attack and mortality in the observation group were significantly lower than those in control group at 12 months after treatment $(\mathrm{p}<0.05)$ (Table IV).

\section{Discussion}

ICVD is a class of diseases characterized by local neurological dysfunction as a result of cerebral blood flow disorders caused by cerebral arterial thrombosis. Ischemic brain tissue can be irreversibly damaged in a short time and cause systemic reactions (9-12). The key point of clinically treating ICVD is to open the occluded vessels as soon as possible, so as to restore blood flow to the brain to improve the patient's prognosis $(13,14)$. Main treatment of ICVD, including thrombolysis, fibrinolysis, anticoagulant, anti-platelet aggregation and other drug 
treatment, usually provide poor treatment outcomes (15). Vascular interventional therapy, including stenosis angioplasty, acute arterial occlusive thrombolysis and recanalization of chronic occlusion arteries, is achieved by unblocking the obstructed blood vessel by placing a stent in the obstructed blood vessel to restore blood flow (16). Interventional therapy, as a new treatment, has the advantages of simple operation, high tolerance and few complications, which can reduce the morbidity and mortality of the disease $(17,18)$.

In this study, rat models of cerebral ischemia were established. Rats were randomly divided into vascular intervention group and aspirin combined with defibrase drug treatment group. The Vs, Vd and neurological deficit of the vascular lesion and the severity of cerebrovascular disease events were compared to explore the therapeutic values of vascular intervention in the treatment of ICVD. All experiments were performed by using scientific and standardized methods with strict internal quality control, so the results had high accuracy and reliability.

Vascular intervention and aspirin combined defibrase were used in the treatment of cerebral ischemia and therapeutic effects were compared. Before treatment, no significant differences in Vs and Vd at vascular lesions were found between the groups. After treatment, Vs and Vd were lower in observation group than those in control group. After treatment, values of Vs and Vd in observation group were significantly reduced, while no significant changes of $\mathrm{Vs}$ and Vd were found in control group. The data suggest that vascular intervention can reduce blood flow resistance to improve ICVD. Wang et al (19) and Singh et al (20) showed that interventional therapy can increase patients' survival and improve their quality of life.

Neurological deficits of rats in observation group and control group were scored before and after 6 and 12 months of treatment. Results showed that NDS score of observation group was lower than that of control group at 6 and 12 months after treatment. NDS score in control group was not significantly changed at 6 and 12 months after treatment compared with pretreatment values, while NDS score in observation group was significantly reduced at 6 and 12 months after treatment compared with pretreatment values. The data show that interventional treatment of blood vessels can improve neurological function in patients, which is consistent with the findings reported by Ha et al (21). Vascular intervention can expand stenosed blood vessels, thereby restoring brain tissue blood supply, promoting the repair of damaged brain regeneration and improving patient's neurological function. Rankine-Mullings et al (22) showed that the cerebral blood flow was improved, and the incidence of neuronal damage in brain tissue decreased after interventional treatment.

This study also found that incidence of cerebral infarction, transient ischemic incidence and mortality in observation group were significantly lower than those of control group at 12 months after treatment, indicating that vascular intervention can reduce the incidence of cerebrovascular disease events. Interventional therapy can restore normal blood flow to ischemic brain tissue, promote recovery of neurological function, and reduce the incidence of cerebrovascular events by implanting scaffolds at stenotic sites of diseased vessels and dilating stenosed vessels (23). Endovascular stents also promote the repair of damaged intima and prevent the shedding of arterial plaque and thrombus formation $(24,25)$.

In conclusion, intracranial vascular interventional therapy can achieve satisfactory outcomes in the treatment of cerebral ischemia, and can effectively improve the patient's neurological function, reduce the incidence of cerebrovascular disease, extend the patient's survival time and improve the quality of life. Therefore, this technique should be popularized.

\section{Acknowledgements}

Not applicable.

\section{Funding}

No funding was received.

\section{Availability of data and materials}

The datasets used and/or analyzed during the present study are available from the corresponding author on reasonable request.

\section{Authors' contributions}

BL conceived the study and wrote the paper. SG and YD interpreted the results. YD revised and finalized the paper. All authors read and approved the final manuscript.

\section{Ethics approval and consent to participate}

The study was approved by the Ethics Committee of The Third People's Hospital of Qingdao (Qingdao, China).

\section{Patient consent for publication}

Not applicable.

\section{Competing interests}

The authors declare that they have no competing interests.

\section{References}

1. Huang YS, Koo M, Chen JC and Hwang JH: The association between tinnitus and the risk of ischemic cerebrovascular disease in young and middle-aged patients: A secondary case-control analysis of a nationwide, population-based health claims database. PLoS One 12: e0187474, 2017.

2. Bondonno CP, Blekkenhorst LC, Prince RL, Ivey KL, Lewis JR, Devine A, Woodman RJ, Lundberg JO, Croft KD, Thompson PL, et al: Association of vegetable nitrate intake with carotid atherosclerosis and ischemic cerebrovascular disease in older women. Stroke 48: 1724-1729, 2017.

3. Lai SW, Lin HF, Lin CL and Liao KF: Long-term effects of pioglitazone on first attack of ischemic cerebrovascular disease in older people with type 2 diabetes: A case-control study in Taiwan. Medicine (Baltimore) 95: e4455, 2016.

4. Wang GQ, Xie YM, Liu H, Zhang Y, Jia PP and Zhuang Y: Drug combination characteristics of Shenxiong glucose injection in treating ischemic cerebrovascular disease in real world. Zhongguo Zhong Yao Za Zhi 42: 2808-2813, 2017 (In Chinese).

5. Ikeda-Sakai Y, Sasaki M and Nakase T: Effects with and without clopidogrel loading treatment for acute ischemic cerebrovascular disease patients: A retrospective cohort study. J Stroke Cerebrovasc Dis 26: 2901-2908, 2017. 
6. Zuo FT, Liu H, Wu HJ, Su N, Liu JQ and Dong AQ: The effectiveness and safety of dual antiplatelet therapy in ischemic cerebrovascular disease with intracranial and extracranial arteriostenosis in Chinese patients: A randomized and controlled trail. Medicine (Baltimore) 96: e5497, 2017.

7. Kurz $\mathrm{T}$ and Thiele $\mathrm{H}$ : Interventional therapy for aortic valve stenosis. MMW Fortschr Med 158: 49-52, 2016 (In German)

8. Longa EZ, Weinstein PR, Carlson S and Cummins R: Reversible middle cerebral artery occlusion without craniectomy in rats. Stroke 20: 84-91, 1989.

9. Qian LH, Li NG, Tang YP, Zhang L, Tang H, Wang ZJ, Liu L, Song SL, Guo JM and Ding AW: Synthesis and bio-activity evaluation of scutellarein as a potent agent for the therapy of ischemic cerebrovascular disease. Int J Mol Sci 12: 8208-8216, 2011.

10. Sharma VK, Tsivgoulis G, Lao AY and Alexandrov AV: Role of transcranial Doppler ultrasonography in evaluation of patients with cerebrovascular disease. Curr Neurol Neurosci Rep 7: 8-20, 2007.

11. Hira RS, Cowart JB, Akeroyd JM, Ramsey DJ, Pokharel Y, Nambi V, Jneid H, Deswal A, Denktas A, Taylor A, et al: Risk factor optimization and guideline-directed medical therapy in US veterans with peripheral arterial and ischemic cerebrovascular disease compared to veterans with coronary heart disease. Am J Cardiol 118: 1144-1149, 2016.

12. Franzone A, Piccolo R, Gargiulo G, Ariotti S, Marino M, Santucci A, Baldo A, Magnani G, Moschovitis A, Windecker S, et al: Prolonged vs short duration of dual antiplatelet therapy after percutaneous coronary intervention in patients with or without peripheral arterial disease: A subgroup analysis of the PRODIGY randomized clinical trial. JAMA Cardiol 1: 795-803, 2016.

13. Della-Morte D, Pacifici F and Rundek T: Genetic susceptibility to cerebrovascular disease. Curr Opin Lipidol 27: 187-195, 2016.

14. Zhou Y, Pan Y, Wu Y, Zhao X, Li H, Wang D, Johnston SC, Liu L, Wang C, Meng X, et al; CHANCE Investigators: Effect of estimated glomerular filtration rate decline on the efficacy and safety of clopidogrel with aspirin in minor stroke or transient ischemic attack: CHANCE substudy (clopidogrel in high-risk patients with acute nondisabling cerebrovascular events). Stroke 47: 2791-2796, 2016

15. Senoo K, Lau YC, Dzeshka M, Lane D, Okumura K and Lip GY: Efficacy and safety of non-vitamin K antagonist oral anticoagulants vs. warfarin in Japanese patients with atrial fibrillation - meta-analysis. Circ J 79: 339-345, 2015.

16. Chen $\mathrm{CH}$, Lin $\mathrm{CL}$ and $\mathrm{Kao} \mathrm{CH}$ : Subtotal gastrectomy with billroth II anastomosis is associated with a low risk of ischemic stroke in peptic ulcer disease patients: A nationwide population-based study. Medicine (Baltimore) 95: e3481, 2016.
17. Menon BK, Sajobi TT, Zhang Y, Rempel JL, Shuaib A, Thornton J, Williams D, Roy D, Poppe AY, Jovin TG, et al: Analysis of workflow and time to treatment on thrombectomy outcome in the ESCAPE randomized controlled trial. Circulation 133: 2279$2286,2016$.

18. Ouyang F, Chen Y, Zhao Y, Dang G, Liang J and Zeng J: Selection of patients and anesthetic types for endovascular treatment in acute ischemic stroke: A meta-analysis of randomized controlled trials. PLoS One 11: e0151210, 2016.

19. Wang S, Jiang J, Qu C, Wang C and Sun Z: Predictive value of serum pregnancy-associated plasma protein A for patients with ischemic cerebrovascular disease. J Clin Lab Anal 31: 22091-22092, 2017.

20. Singh AG, Crowson CS, Singh S, Denis M, Davis P, MaraditKremers H, Matteson EL and Chowdhary VR: Risk of cerebrovascular accidents and ischemic heart disease in cutaneous lupus erythematosus: A population-based cohort study. Arthritis Care Res (Hoboken) 68: 1664-1670, 2016.

21. Ha M, Choi CH, Lee JI, Cha SH, Lee SW and Ko JK: The efficacy of single barrel superficial temporal artery-middle cerebral artery bypass in treatment of adult patients with ischemic-type moyamoya disease. J Cerebrovasc Endovasc Neurosurg 18: 239-246, 2016

22. Rankine-Mullings AE, Little CR, Reid ME, Soares DP, Taylor-Bryan C, Knight-Madden JM, Stuber SE, Badaloo AV, Aldred K, Wisdom-Phipps ME, et al: EXpanding treatment for existing neurological disease (EXTEND): An open-label phase II clinical trial of hydroxyurea treatment in sickle cell anemia. JMIR Res Protoc 5: e185, 2016.

23. Hira RS, Cowart JB, Akeroyd JM, Ramsey DJ, Pokharel Y, Nambi V, Jneid H, Deswal A, Denktas A, Taylor A, et al: Risk factor optimization and guideline-directed medical therapy in US veterans with peripheral arterial and ischemic cerebrovascular disease compared to veterans with coronary heart disease. Am J Cardiol 118: 1144-1149, 2016.

24. Nussbaum ES and Erickson DL: Extracranial-intracranial bypass for ischemic cerebrovascular disease refractory to maximal medical therapy. Neurosurgery 46: 37-42, 2000.

25. Yamazaki M, Ohnishi T, Hosokawa K, Yamaguchi K, Yoneyama T, Kawashima A, Okada Y, Kitagawa K and Uchiyama S: Measurement of residual platelet thrombogenicity under arterial shear conditions in cerebrovascular disease patients receiving antiplatelet therapy. J Thromb Haemost 14: 1788-1797, 2016.

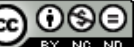

This work is licensed under a Creative Commons Attribution-NonCommercial-NoDerivatives 4.0 International (CC BY-NC-ND 4.0) License. 